\title{
A homolog of glyceraldehyde-3-phosphate dehydrogenase from \\ Riemerella anatipestifer is an extracellular protein and exhibits biological activity*
}

\author{
Ji-ye GAO, Cui-lian YE, Li-li ZHU, Zhi-ying TIAN, Zhi-bang YANG ${ }^{\dagger t}$ \\ (Department of Basic Medicine, Chongqing Medical University, Chongqing 400016, China) \\ †E-mail: dryangfm365@sina.com \\ Received Jan. 24, 2014; Revision accepted May 29, 2014; Crosschecked Aug. 21, 2014
}

\begin{abstract}
Riemerella anatipestifer is the causative agent of septicemia anserum exsudativa in ducks. Its pathogenesis and virulence factors are still unclear. The glycolytic enzyme, glyceraldehyde-3-phosphate dehydrogenase (GAPDH), an anchorless and multifunctional protein on the surface of several pathogenic microorganisms, is involved in virulence and adhesion. Whether homologs of GAPDH exist, and display similar characteristics in $R$. anatipestifer (RaGAPDH) has not been determined. In our research, the RaGAPDH activity from various $R$. anatipestifer isolates was confirmed. Twenty-two gapdh genes from genomic DNA of $R$. anatipestifer isolates were cloned and sequenced for phylogenetic analysis. The distribution of RaGAPDH in R. anatipestifer CZ2 strain was confirmed by antisera to recombinant RaGAPDH. The ability of purified RaGAPDH to bind host proteins was analyzed by solid-phase ligandbinding assay. Results revealed that all $R$. anatipestifer isolates showed different levels of GAPDH activity except four strains, which contained a gapdh-like gene. The gapdh of $R$. anatipestifer, which is located phylogenetically in the same branch as enterohemorrhagic Escherichia coli (EHEC), belonged to class I GAPDH, and encoded a 36.7-kDa protein. All RaGAPDH-encoding gene sequences from field isolates of $R$. anatipestifer displayed $100 \%$ homology. The RaGAPDH localized on the extracellular membrane of several $R$. anatipestifer strains. Further, it was released into the culture medium, and exhibited GAPDH enzyme activity. We also confirmed the binding of RaGAPDH to plasminogen and fibrinogen. These results demonstrated that GAPDH was present in $R$. anatipestifer, although not in all strains, and that RaGAPDH might contribute to the microorganism's virulence.
\end{abstract}

Key words: Riemerella anatipestifer, Glyceraldehyde-3-phosphate dehydrogenase (GAPDH), Extracellular protein doi:10.1631/jzus.B1400023

Document code: A

CLC number: Q936; S85

\section{Introduction}

Riemerella anatipestifer is a Gram-negative, non-motile, non-spore-forming, rod-shaped bacterium, belonging to the family Flavobacteriaceae in the ribosomal RNA (rRNA) superfamily V based on $16 \mathrm{~S}$ rRNA gene sequence analysis (Segers et al., 1993; Subramaniam et al., 1997; Tsai et al., 2005). It causes an acute or chronic primary septicemic disease in domestic ducks, geese, turkeys, and other wild birds,

\footnotetext{
${ }^{\ddagger}$ Corresponding author

* Project supported by the Fundamental Research Funds of the Central Universities of China (No. XDJK2013C009)

(C) Zhejiang University and Springer-Verlag Berlin Heidelberg 2014
}

characterized by fibrinous pericarditis, perihepatitis, air sacculitis, caseous salpingitis, meningitis, and accounts for major economic losses to the duckrearing industry (Leavitt and Ayroud, 1997). Currently, 16 serotypes of $R$. anatipestifer have been isolated and identified in China among the $21 R$. anatipestifer serotypes described to date (Pathanasophon et al., 1995; 2002; Subramaniam et al., 2000). Among the known serotypes of $R$. anatipestifer, there are huge variations in the virulence between serotypes and between different strains even within the same serotype (Subramaniam et al., 2000). Although four genome sequences of $R$. anatipestifer of strains ATCC 11845 (Mavromatis et al., 2011), RA-GD 
(Yuan et al., 2011), RA-YM (Zhou et al., 2010), and RA-SG (Yuan et al., 2013) have been reported, little is known about the molecular basis of the pathogenicity of $R$. anatipestifer infection, and so far, few virulence factors have been characterized other than virulence-associated protein D (VapD) (Chang et al., 1998), CAMP cohemolysin (Crasta et al., 2002), and outer membrane protein A (OmpA) (Hu et al., 2011).

Glyceraldehyde-3-phosphate dehydrogenase (GAPDH) is a classical glycolytic enzyme converting D-glyceraldehyde-3-phosphate into 1,3-diphosphoglycerate. Members of the GAPDH family are classified into the ubiquitous class I enzymes that utilize nicotinamide adenine dinucleotide $\left(\mathrm{NAD}^{+}\right)$(EC 1.2.1.12), $\mathrm{NADP}^{+}$(EC 1.2.1.13 or 1.2.1.59), class II of archaeal $\mathrm{NAD}(\mathrm{P})^{+}$-dependent GAPDHs, and class III bifunctional enzymes (erythose-4-phosphate dehydrogenase/GAPDH) that are prevalent among $\gamma$-proteobacteria (Figge et al., 1999). In addition to its classical glycolytic roles, GAPDH is involved in a number of fundamental cellular pathways such as maintenance of DNA integrity, intracellular membrane trafficking, histone-gene regulation, receptormediated cell signaling, protection of telomeric DNA, post-transcriptional gene regulation, autophagy, apoptosis, and oxidative stress response; all these depend on the ability of GAPDH to modify its subcellular localization (Sirover, 2011). Thus, GAPDH is widely used as a model protein or control in gene regulation and catalytic-mechanism-related studies, as well as a standard in Northern- and Western-blots, because of its highly conserved structure across species (Zheng et al., 2003). Recent studies have demonstrated that GAPDH is presented on the surface of several microbial pathogens such as Streptococcus agalactiae (Seifert et al., 2003), S. pneumonia (Ling et al., 2004), Listeria monocytogenes (Schaumburg et al., 2004), enterohemorrhagic Escherichia coli (EHEC), and enteropathogenic Escherichia coli (EPEC) (Egea et al., 2007), and may facilitate their colonization and invasion of host tissues by interacting directly with host-soluble proteins and surface ligands (Kenny and Finlay, 1995; Pancholi and Chhatwal, 2003). Its roles are also implicated in some pathogenic microorganisms like Candida albicans, Schistosoma bovis, and Mycoplasma genitalium (Alvarez et al., 2007). Furthermore, secreted GAPDH by $S$. pyogenes and enteropathogenic $E$. coli strains
(Kenny and Finlay, 1995; Eichenbaum et al., 1996; Aguilera et al., 2012) plays a role in their adhesion and virulence (Modun and Williams, 1999; Daubenberger et al., 2000; Modun et al., 2000; Parker and Bermudez, 2000; Nagradova, 2001; Schaumburg et al., 2004; Jin et al., 2005; Alvarez et al., 2007; Colell et al., 2007; Egea et al., 2007; Tunio et al., 2010). Although one copy homolog of GAPDHencoding gene was identified in the genomes of $R$. anatipestifer strains ATCC11845, RA-GD, RA-CH-1, and RA-SG in our previous bioinformatics analysis, it remains to be addressed whether GAPDH from these strains is in the form of an extracellular protein and displays similar characteristics as reported in other pathogens.

In the present study, the genes of GAPDH from various $R$. anatipestifer (RaGAPDH) isolates were sequenced for phylogenetic analysis. We generated antisera to recombinant RaGAPDH of $R$. anatipestifer CZ2 strain, and identified the biologically active RaGAPDH secreted in the culture of $R$. anatipestifer.

\section{Materials and methods}

\subsection{Bacterial strains, culture conditions, and ge-} nomic DNA extraction

Twenty-two $R$. anatipestifer field isolates from Sichuan Province and Chongqing Municipality in China are described in Table 1. The strains of $R$. anatipestifer were grown on trypticase soy agar (OXOID Ltd., UK) or cultured in tryptic soybean broth (TSB; OXOID Ltd., UK) at $37{ }^{\circ} \mathrm{C}$ in an atmosphere containing $5 \% \mathrm{CO}_{2}$. E. coli strains DH5 $\alpha$ and BL21 $\left(\lambda \mathrm{DE}_{3}\right)$, used as hosts for cloning and expression of gapdh gene from $R$. anatipestifer, respectively, were grown routinely on Luria broth (LB) agar or in LB broth at $37^{\circ} \mathrm{C}$. Genomic DNA (gDNA) was extracted from all the field isolates using the MiniBEST bacterial gDNA extraction kit V.2.0 (TaKaRa Biotech Co., Ltd., China). The concentration and quality of the gDNA were established by optical density measurements at $260 / 280 \mathrm{~nm}$.

\subsection{Isolation of secreted proteins in culture medium}

Soluble proteins in $R$. anatipestifer culture medium were isolated as previously described (Egea $\mathrm{et} \mathrm{al.,}$ 
2007) with some modification. Briefly, exponential phase cultures in TSB were diluted 1:50 in the indicated culture media and incubated at $37{ }^{\circ} \mathrm{C}$ in a $5 \%$ $\mathrm{CO}_{2}$ atmosphere. At different times, the bacterial cells were removed by centrifugation $\left(5000 \mathrm{~g}, 10 \mathrm{~min}, 4^{\circ} \mathrm{C}\right)$ and the supernatant removed. The proteins in the filtrate were precipitated with $15 \%$ trichloroacetic acid (TCA) by incubation on ice for $3 \mathrm{~h}$. The protein pellet was washed in $90 \%$ ice-cold acetone, air-dried, and suspended in loading buffer for sodium dodecyl sulfatepolyacrylamide gel electrophoresis (SDS-PAGE).

Table 1 Twenty-two $R$. anatipestifer strains

\begin{tabular}{|c|c|c|c|c|}
\hline Isolate & $\begin{array}{l}\text { Year of } \\
\text { isolation }\end{array}$ & Serotype & $\begin{array}{l}\text { Extracellular } \\
\text { GAPDH } \\
\text { activity }^{*}\end{array}$ & $\begin{array}{l}\text { Sequencing } \\
\text { result }(\mathrm{bp})\end{array}$ \\
\hline $\mathrm{AF}^{\mathrm{a}}$ & 2001 & 2 & $0.536 \pm 0.038$ & 996 \\
\hline $\mathrm{CZ}^{\mathrm{a}}{ }^{\mathrm{a}}$ & 2007 & 1 & $0.678 \pm 0.033$ & 990 \\
\hline $\mathrm{CZ2}^{\mathrm{a}}$ & 2003 & 1 & $0.484 \pm 0.019$ & 992 \\
\hline $\mathrm{DX} 1^{\mathrm{a}}$ & 2013 & & $0.009 \pm 0.002$ & \\
\hline DY $1109^{a}$ & 2011 & & $0.215 \pm 0.012$ & 994 \\
\hline DY $1202^{a}$ & 2012 & & $0.636 \pm 0.033$ & 998 \\
\hline DY $1210^{\mathrm{a}}$ & 2012 & & $0.478 \pm 0.035$ & 989 \\
\hline $\mathrm{DZ} 1108^{\mathrm{a}}$ & 2011 & & $0.628 \pm 0.024$ & 987 \\
\hline $\mathrm{DZ1209^{ \textrm {a } }}$ & 2012 & & $0.573 \pm 0.030$ & 964 \\
\hline $\mathrm{DZ} 1212^{\mathrm{a}}$ & 2012 & & $0.341 \pm 0.032$ & 991 \\
\hline $\mathrm{gHN} 1210^{\mathrm{b}}$ & 2012 & 2 & $0.371 \pm 0.027$ & 992 \\
\hline gXJY12b & 2012 & & $0.545 \pm 0.027$ & 990 \\
\hline $\mathrm{JJ} 1212^{\mathrm{a}}$ & 2012 & & $0.309 \pm 0.025$ & 991 \\
\hline $\mathrm{LS}^{\mathrm{a}}$ & 2004 & & $0.587 \pm 0.035$ & 994 \\
\hline $\mathrm{MY}^{\mathrm{a}}$ & 2002 & & $0.427 \pm 0.024$ & 996 \\
\hline PN1209 & 2012 & & $0.004 \pm 0.003$ & \\
\hline $\mathrm{RL}^{\mathrm{a}}$ & 2009 & & $0.578 \pm 0.034$ & 998 \\
\hline $\mathrm{SC} 12^{\mathrm{a}}$ & 2012 & 1 & $0.157 \pm 0.039$ & 998 \\
\hline $\mathrm{TL}^{\mathrm{a}}$ & 2010 & & $0.624 \pm 0.038$ & 991 \\
\hline $\mathrm{WS} 1210^{\mathrm{a}}$ & 2012 & & $0.414 \pm 0.037$ & 1001 \\
\hline $\mathrm{YCl}^{\mathrm{a}}$ & 2012 & & $0.006 \pm 0.002$ & \\
\hline $\mathrm{YC}^{\mathrm{a}}$ & 2012 & & $0.003 \pm 0.002$ & \\
\hline
\end{tabular}

${ }^{\mathrm{a}}$ Isolated from duck; ${ }^{\mathrm{b}}$ Isolated from goose. ${ }^{*}$ Data are shown as mean \pm standard error (SE) $(n=3)$ of the absorbance at $340 \mathrm{~nm}$ determined by the method described in Section 2.3

\subsection{Enzyme activity}

The RaGAPDH activity on the bacterial cell surface was measured according to Egea et al. (2007) with some modification. Briefly, bacterial cells were centrifuged at $4500 \mathrm{~g}$ for $5 \mathrm{~min}$ and the pellets were washed twice in $50 \mathrm{mmol} / \mathrm{L} \mathrm{Na}_{2} \mathrm{HPO}_{4}, \mathrm{pH} 7.5$, and then resuspended in the same buffer. Bacterial suspensions of $4 \times 10^{8} \mathrm{CFU}$ (colony-forming unit) $/ 200 \mu \mathrm{l}$ were incubated with $14 \mu$ l glyceraldehyde-3-phosphate $(50 \mathrm{mg} / \mathrm{ml}), 200 \mu \mathrm{l} \mathrm{NAD}{ }^{+}(10 \mathrm{mmol} / \mathrm{L})$, and assay buffer $(40 \mathrm{mmol} / \mathrm{L}$ triethanolamine, $50 \mathrm{mmol} / \mathrm{L}$ $\mathrm{Na}_{2} \mathrm{HPO}_{4}$, and $5 \mathrm{mmol} / \mathrm{L}$ EDTA; $\mathrm{pH}$ 8.6) in a final volume of $2 \mathrm{ml}$. After $15 \mathrm{~min}$ at $30^{\circ} \mathrm{C}$, cells were removed by centrifugation and the supernatant was examined for the presence of nicotinamide adenine dinucleotide (NADH) by measuring the absorbance at $340 \mathrm{~nm}$. Control reactions were used without bacterial cells or glyceraldehyde-3-phosphate.

The GAPDH activity of the secreted protein mixture from three strains (two showed positive GAPDH activity on the surface of bacterial cells) was measured, following the increase in absorbance at $340 \mathrm{~nm}$ by the formation of NADH (Egea et al., 2007). Briefly, supernatants from $R$. anatipestifer bacterial cultures were passed through a $0.22-\mu \mathrm{m}$ filter and precipitated with $95 \%\left(\mathrm{NH}_{4}\right)_{2} \mathrm{SO}_{4}$. The protein pellets were resuspended in a buffer solution $(50 \mathrm{mmol} / \mathrm{L}$ $\mathrm{Na}_{2} \mathrm{HPO}_{4}, \mathrm{pH} 7.5$ ) and used for determining the enzymatic activity of secreted GAPDH.

\subsection{Identification of the gapdh in R. anatipestifer}

The GAPDH amino acid sequences of 13 species obtained from GenBank, including Xenopus laevis (P51469), Gallus gallus (P00356), E. coli (P0A9B4), Homo sapiens (P0034), Drosophila melanogaster (Q01597), Trypanosoma brucei (P00097), Plasmodium falciparum (AF030440), Gonyaulax polyedra (AF028562), Leishmania mexicana (Q01558), Candida albicans (Q92211), and three R. anatipestifer strains (ATCC11845 (CP003388), RA-CH-1 (NC018609), and RA-GD (CP002562)), were aligned and analyzed by the clustal W method of the MegAlign II program (DNAStar, Madison, WI). Two degenerate primers RaGAPDH1 and RaGAPDH2 (Table 2) based on the conserved region of the GAPDH amino acids were designed and used for polymerase chain reaction (PCR) amplification. The PCR amplifications were performed using the above primers and the Taq ${ }^{\mathrm{TM}}$ System (TaKaRa) under the following conditions: 30 cycles of denaturation $\left(95^{\circ} \mathrm{C}, 1 \mathrm{~min}\right)$, annealing $\left(52^{\circ} \mathrm{C}, 1 \mathrm{~min}\right)$, and extension $\left(72{ }^{\circ} \mathrm{C}, 1 \mathrm{~min}\right)$. The first round PCR products (diluted 200 times) were amplified by the second PCR in a $50-\mu 1$ reaction mixture under the same conditions. All the second round PCR products were purified using the MiniBest Agarose Gel DNA Extraction Kit Ver. 4.0 (TaKaRa) and inserted in the pMD19 ${ }^{\mathrm{R}}-\mathrm{T}$ (TaKaRa) cloning vector, according to the manufacturer's instructions. 
Table 2 Primers used in this study

\begin{tabular}{ll}
\hline \multicolumn{1}{c}{ Primer } & \multicolumn{1}{c}{ Sequence } \\
\hline Primers for cloning gapdh & \\
RaGAPDH1 & 5'-GGNTTYGGNCGNATHGGNCGN-3' (N=A/G/C/T,Y=C/T,H=A/C/T) \\
RaGAPDH2 & 5'-YTCRTTRTCRTACCANGA-3' (Y=C/T, R=A/G, N=A/G/C/T) \\
Primers for genomic walking & \\
uSP1 & 5'-CGCACCTACTTCATTCCATTTTAGATTG-3' \\
uSP2 & 5'-CTCCATCAAATTTTCCGTGAACAGA-3' \\
uSP3 & 5'-CTAAACTTCCGATTCTTCCGAAACCG-3' \\
dSP1 & 5'-AGGTATGTCTTTCCGTGTACCAACG-3' \\
dSP2 & 5'-GGGAGAACTTAAAGGTATCCTTGGT-3' \\
dSP3 & 5'-AGTATCTTGGTACGATAACGAAACAGG-3' \\
Primers for expression & \\
Egap1F & 5'-GAGGAATTCATGTCAACAATCAAAG-3' (EcoRI site underlined) \\
Egap1R & 5'-ATTCTCGAGTAAAGAAGCAGAGTGT-3' (XhoI site underlined) \\
\hline
\end{tabular}

Plasmid DNA containing gapdh of $R$. anatipestifer was purified and sequenced at BGI Biotech Co., Ltd., China. Comparison and alignments of deduced amino acid sequences of GAPDH from $R$. anatipestifer strains were conducted using SeqMan program (DNAStar, Madison, WI). Clustal multiple alignment algorithms were used to calculate the similarity and divergence of the sequences. The phylogenetic tree was generated using the molecular evolutionary genetics analysis (MEGA) program V5.2 with 10000 bootstrap replicates. Sixty-six other amino acid sequences (Table 3 ) obtained from GenBank were also included in the analysis.

\subsection{Genomic walking of flanking sequences of gapdh gene}

A genome walking kit (TaKaRa) was used to clone the left and right flanking sequences of the gapdh gene in $R$. anatipestifer CZ2 strain according to the manufacturer's instructions. The gapdhspecific primers (Table 2), which were designed on the basis of the nucleotide sequence of the target fragments and used for the 1st, 2nd, and 3rd nested PCR, and the sense primer AP3 provided with the kit, were used to amplify the 5'- and 3 '-terminus. The PCR products were cloned into the $\mathrm{pMD} 19^{\mathrm{R}}-\mathrm{T}$ vector (TaKaRa). DNA sequencing was performed in the Beijing BGI-GBI Biotech Co., Ltd., China.

\subsection{Preparation of mouse antiserum against re- combinant RaGAPDH}

Open reading frame (ORF) of RaGAPDH was amplified from gDNA of $R$. anatipestifer CZ2 strain by PCR using primers Egap1F and Egap1R containing EcoRI and XhoI sites, respectively. The products were digested with EcoRI and XhoI, purified and then cloned in frame into the bacterial expression vector pET-28a $(+)$ (Novagen). The recombinant plasmid was sequenced to confirm that the RaGAPDH insert was in the proper reading frame and transformed into the competent E. coli BL21 (DE3) cells. The cells harboring the recombinant plasmid were grown at $37{ }^{\circ} \mathrm{C}$ in a rotary shaker at $160 \mathrm{r} / \mathrm{min}$, in $100 \mathrm{ml} \mathrm{LB}$ broth supplemented with $100 \mu \mathrm{g} / \mathrm{ml}$ ampicillin. At the point when the absorbance at $600 \mathrm{~nm}\left(A_{600}\right)$ reached $0.6,0.5 \mathrm{mmol} / \mathrm{L}$ isopropyl thiogalactoside (IPTG) was added to induce the recombinant protein expression. The cells were induced at $37^{\circ} \mathrm{C}$ for $3 \mathrm{~h}$ and total cell lysates were prepared and analyzed by SDSPAGE. The expressing recombinant protein was harvested and purified using Magnehis ${ }^{\mathrm{TM}}$ protein purification system (Promega, USA) according to the manufacturer's instructions. Antisera against recombinant RaGAPDH were obtained by immunization of 4-week-old female BALB/c mice subcutaneously injected four times at 2-week intervals with $30 \mu \mathrm{g}$ of protein emulsified in Freund's complete (first immunization only) or incomplete adjuvant. Mice sera were collected at $7 \mathrm{~d}$ after the fourth immunization and antibody titer was determined.

\subsection{Western-blot analysis}

For Western-blot analysis, recombinant RaGAPDH or secreted proteins in eight $R$. anatipestifer culture media were electrophoresed on $10 \%$ gels and transferred to a polyvinylidene difluoride 
Table 3 Species and identification numbers of origin in GenBank for the sequences used in this study

\begin{tabular}{|c|c|c|c|}
\hline Species/strain & Sequence ID & Species/strain & Sequence ID \\
\hline Ginkgo biloba & Q39769 & Galdieria sulphuraria & AJ012286 \\
\hline Methanothermus fervidus & ADP77079 & Ralstonia solanacearum & NP520870 \\
\hline Dirofilaria immitis & AFL46382 & Anabaena variabilis & YP321014 \\
\hline Mycoplasma agalactiae & YP003303052 & Chlamydophila caviae & NP828990 \\
\hline Mycoplasma mycoides subsp. & YP004400344 & Thermotoga maritime & NP228497 \\
\hline Streptococcus pneumoniae & AAK76079 & Deinococcus geothermalis & YP604599 \\
\hline Streptococcus dysgalactiae & CAA66377 & Physicomitrella patens & P34923 \\
\hline Streptococcus agalactiae & AF3384161 & Dianthus caryophyllus & P34921 \\
\hline Streptococcus pyogenes & AAK33348 & Atriplex nummularia & P34783 \\
\hline Streptococcus suis & AAN86058 & Aspergillus niger & Q12553 \\
\hline Chondrus crispus & X73033 & R. anatipestifer $\mathrm{RA}-\mathrm{CH}-1$ & NC018609 \\
\hline Drosophila melanogaster & NM080352 & Caenorhabditis elegans & NM076134 \\
\hline Cyanophora paradoxa & AJ313316 & Karenia mikimotoi & AB164183 \\
\hline Bacteroides fragilis & YP098251 & Heterosigma akashiwo & AF319448 \\
\hline Synechocystis sp. PCC 6803 & NP440929 & Guillardia theta & CPU40032 \\
\hline Treponema denticola & NP972094 & Candida albicans & XM714816 \\
\hline Thermus thermophilus & YP004524; & Pyrenomonas salina & PSU40033 \\
\hline Geobacillus kaustophilus & YP148579 & Agrobacterium fabrum & AAK89669 \\
\hline Parastagonospora nodorum & $\mathrm{AJ} 271155$ & Candida albicans & Q92211 \\
\hline Paracoccidioides brasiliensis & AF396657 & Drosophila melanogaster & Q01597 \\
\hline Gallus gallus & P00356 & Ustilago maydis & P09317 \\
\hline Escherichia coli $\mathrm{O} 157$ & P06977 & Neurospora crassa & XM951884 \\
\hline R. anatipestifer ATCC 11845 & СР003388 & Ustilago maydis & X07879 \\
\hline Trypanosoma cruzi & XM814806 & Pyropia yezoensis & AY273819 \\
\hline Saccharomyces cerevisiae & NM001181485 & Escherichia coli CFT073 & NP753744 \\
\hline Schizosaccharomyces pombe & NM001021142 & Thermosynechococcus elongatus & NP680834 \\
\hline Pseudomonas aeruginosa & NP249242 & Leptospira interrogans serovar & NP711885 \\
\hline Moorella thermoacetica & YP429140 & Corynebacterium diphtheriae & NP939663 \\
\hline Bacillus halodurans & NP244015 & Thermobifida fusca & YP290073 \\
\hline Leishmania mexicana & Q01558 & Nicotiana tabacum & CAB39974 \\
\hline Gonyaulax polyedra & AF028562 & Xenopus laevis & P51469 \\
\hline Plasmodium falciparum & AF030440 & Pinus sylvestris & P34924 \\
\hline Trypanosoma brucei & P00097 & Homo sapiens & P0034 \\
\hline Caenorhabditis elegans & NM076134 & R. anatipestifer RA-GD & $\mathrm{CP} 002562$ \\
\hline Phaffia rhodozyma & AF006483 & & \\
\hline
\end{tabular}

(PVDF) membrane using a Bio-Rad mini Trans-Blot apparatus. The membrane was blocked and then incubated with anti-recombinant RaGAPDH sera (500× dilution in blocking solution) or with anti-OmpA sera (500× dilution in blocking solution) for $16 \mathrm{~h}$ at $4{ }^{\circ} \mathrm{C}$. After several washing steps, the membrane was incubated with $1000 \times$ dilution of a rabbit anti-mouse $\mathrm{IgG}$ horseradish peroxidase (HP)-conjugated $\mathrm{IgG}$ for $2 \mathrm{~h}$. Blots were developed using the enhanced chemiluminescence (ECL) plus Western-blot detection system (Chimi DOX-XRS, Bio-Rad, US). In this experiment, $\mathrm{AF}, \mathrm{CZ1}, \mathrm{CZ2}$, gHN1210, and $\mathrm{SC} 12$ were selected to detect the secreted RaGAPDH because serotypes 1 and 2 are two of the most prevalent serotypes in China.

\subsection{Solid-phase ligand-binding assay}

The binding of RaGAPDH to host proteins (including actin, plasminogen, fibrinogen, and fibronectin) was determined according to Egea et al. (2007). 
Briefly, actin, plasminogen, fibrinogen, or fibronectin ( $5 \mu \mathrm{g} / \mathrm{ml})$ was bound to the wells $(100 \mu \mathrm{l} /$ well $)$ of a 96-well high-binding microtiter plate for 9-h incubation at room temperature. Positive and negative control wells coated with RaGAPDH and $0.5 \%$ gelatin, respectively, were prepared in parallel. After 12-h incubation with Tris-buffered saline (TBS) blocking buffer (containing 1\% gelatin) and then 3 -h reaction with purified RaGAPDH $(0.125-2.500 \mu \mathrm{g} / \mathrm{ml}$, $100 \mu \mathrm{l} /$ well), the plates were washed three times in TBS containing $0.05 \%$ Tween-20 and once in TBS buffer. The amount of RaGAPDH bound to host proteins was determined spectrophotometrically (492 nm) in an enzyme-linked immunosorbent assay (ELISA)based assay using anti-recombinant-RaGAPDH sera (1:500) followed by peroxidase-labelled rabbit antimouse antibody $(1: 1000)$ and $o$-phenylenediamine as chomogen. The specificity of the absorbance values was assessed in ELISA assays in which incubation with RaGAPDH was omitted. The background values in the negative control obtained with the indicated antibody dilutions were less than 0.05 for all proteins tested, but they were nevertheless subtracted from the test values to obtain specific absorbance.

\section{Results}

\subsection{Secreted protein fraction and bacterial sur- face of $\boldsymbol{R}$. anatipestifer exhibiting GAPDH activity}

To determine the GAPDH activity on the bacterial cell surface, suspensions of whole cells were used as the enzymatic source and resulted in an increase in absorbance at $340 \mathrm{~nm}$ due to the NADH formation. Eighteen of $22 R$. anatipestifer isolate suspensions (the exceptions were DX1, PN1209, YC1, and YC2 strains) co-catalyze the oxidative phosphorylation of D-glyceraldehyde-3-phosphate to 1,3-diphosphoglycerate in the presence of $\mathrm{NAD}^{+}$(Fig. 1a). The presence of secreted RaGAPDH in the culture medium of $R$. anatipestifer $\mathrm{CZ2}$, SC12, and YC1 strains was confirmed by assaying its catalytic activity. To this end, cell-free supernatants at different time intervals were concentrated by TCA and analyzed for GAPDH activity. Supernatants from cultures of CZ2 and $\mathrm{SC} 12$ showed a significant NADH formation coupled to glyceraldehyde-3-phosphate oxidation, while YC1 did not display GAPDH activity (Fig. 1b).

\subsection{RaGAPDH nucleotide sequence and homology}

PCR of gDNA from 18 strains of $R$. anatipestifer with RaGAPDH1 and RaGAPDH2 primers homologous to conserved regions of 13 GAPDH amino acid sequences obtained from GenBank produced a single amplicon of about $1000 \mathrm{bp}$, while the other two stains, PN1209 and DX1, produced one or two amplicons of less than $1000 \mathrm{bp}$; YC1 and YC2 strains did not produce any amplicon (Fig. 2). The products were sequenced and deduced amino acid sequences were compared with the other 69 GAPDH sequences retrieved from GenBank. A phylogenetic tree (Fig. 3) was built using the MEGA5.2 program to determine the origin and identity of the RaGAPDH. The RaGAPDH was localized on the same branch as EHEC, belonging to the class I of bacteria GAPDH (EC 1.2.1.12). Fig. 3 also shows that the origin of RaGAPDH was monophyletic.

The complete sequence of the RaGAPDHcoding gene in $R$. anatipestifer CZ2 strain was identified by genomic walking. The nucleotide sequence predicts an ORF of 334 amino acids, with a predicted molecular mass of approximately $36.7 \mathrm{kDa}$. Hydrophilic analysis of the RaGAPDH protein did not indicate the presence of any transmembrane spanning regions or a signal sequence. Despite considerable divergence from other characterized GAPDH molecules, the RaGAPDH is also composed of two basic domains common to members of the GAPDH family of enzymes: the $\mathrm{NAD}^{+}$-binding domain (residues 4-152 amino acids) and the catalytic domain (residues 157-334 amino acids).

\subsection{Antigenic nature of recombinant RaGAPDH}

To detect and characterize the extracellular RaGAPDH, we were interested in the expression of $\mathrm{RaGAPDH}$ and the generation of specific polyclonal antibodies. After several attempts to determine the optimal conditions for RaGAPDH expression in $E$. coli BL21 (DE3), a high level of expression of recombinant RaGAPDH was obtained by adding $0.5 \mathrm{mmol} / \mathrm{L}$ of IPTG and incubating the culture at $37^{\circ} \mathrm{C}$ for $3 \mathrm{~h}$. The expressed protein was analyzed by SDS-PAGE and Western-blot for purity and homogeneity. As demonstrated in Fig. 4a, a protein band of a subunit of approximately $53.7 \mathrm{kDa}$ was detected after protein staining with Coomassie blue. The 


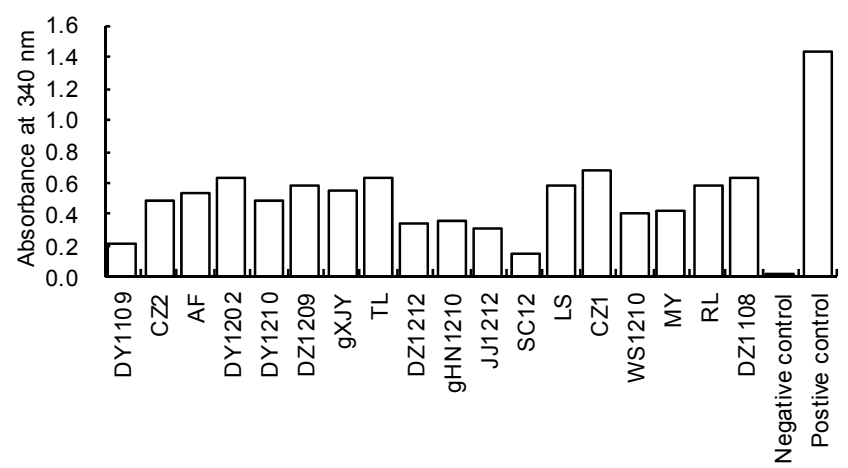

(a)

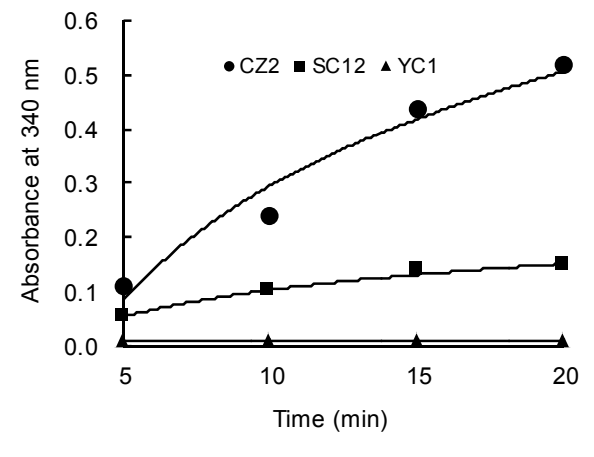

(b)

Fig. 1 Extracellular GAPDH activity of whole cells (a) and cell-free supernatants (b)

The GAPDH activity was measured by monitoring the NADH formation coupled to the conversion of glyceraldehyde3-phosphate to 1,3-bisphosphoglycerate (absorbance at $340 \mathrm{~nm}$ ) at different time intervals or as an endpoint reading after 15 min of incubation. (a) GAPDH activity on the surface of bacterial cells. The GAPDH activity was determined at $30{ }^{\circ} \mathrm{C}$ by measuring the absorbance at $340 \mathrm{~nm}$ as an endpoint reading of the supernatant obtained from whole cells $\left(4 \times 10^{8} \mathrm{CFU} / 200 \mu \mathrm{l}\right)$ after $15 \mathrm{~min}$ of incubation in $2 \mathrm{ml}$ of the assay mixture. Data are presented as the mean of three independent experiments. Negative control reactions were performed in the absence of glyceraldehyde-3-phosphate. (b) Cells of $R$. anatipestifer strains $\mathrm{CZ2}, \mathrm{SC12}$, and $\mathrm{YC} 1$ were collected by centrifugation, and the cell-free supernatants were processed by TCA as described in Section 2.3

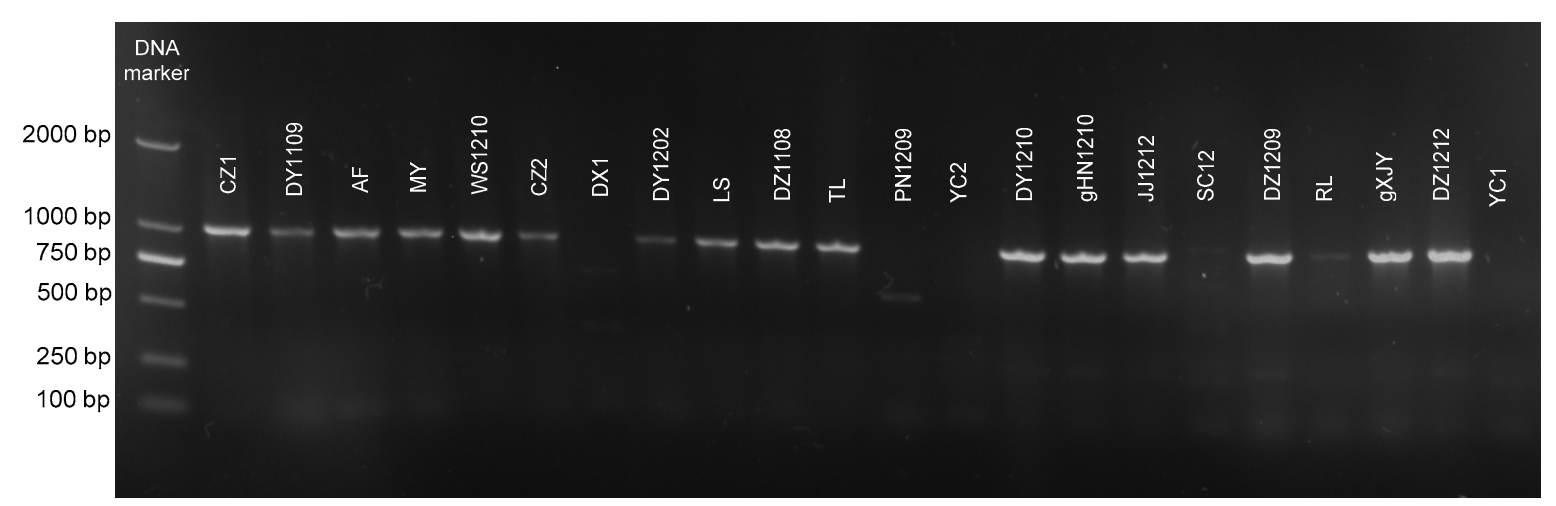

Fig. 2 Identification of a homolog of GAPDH from R.anatipestifer field isolates by PCR

Lane M: DNA molecular weight marker; Lanes CZ1 to YC1: twenty-two strains of R.anatipestifer

recombinant RaGAPDH runs slightly higher than native RaGAPDH, which could be explained by the additional $17 \mathrm{kDa}$ tag-protein not present in the native sequence (Fig. 4c).

\subsection{Identification of RaGAPDH as an extracellu- lar protein in $R$. anatipestifer culture}

The proteins secreted by exponential phase cultures of $R$. anatipestifer grown in TSB were extracted and used for immunoblotting analysis. The extracellular proteins were detected in supernatants of $R$. anatipestifer $\mathrm{AF}, \mathrm{CZ1}, \mathrm{CZ2}, \mathrm{gHN} 1210, \mathrm{SC} 12$,
PN1209, DX1, and YC1 strains (Fig. 4b). To exclude the possibility that the presence of RaGAPDH in the culture medium was due to the contamination from cell lysis, immunoblots were run in parallel using antibodies against the OmpA of $R$. anatipestifer. No extracellular specific band was detected for this control protein in the supernatant cultures of the three strains. The results indicated that the presence of RaGAPDH in the supernatant of exponentially growing bacteria was attributable to a secretion process rather than cell lysis. 
Fig. 3 Phylogenetic tree based on the deduced amino acid sequences of PCR products amplified from gapdh of $R$. anatipestifer isolates

Numbers at branching points represent percentage of 10000 bootstrap values calculated by the MEGA program with the Poisson correction distance optional for amino acid sequences. The scale bar indicates the number of amino acid substitutions per site
0.2

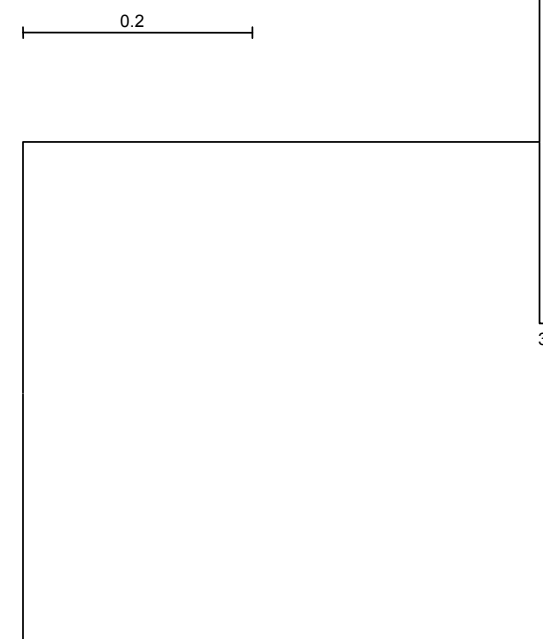

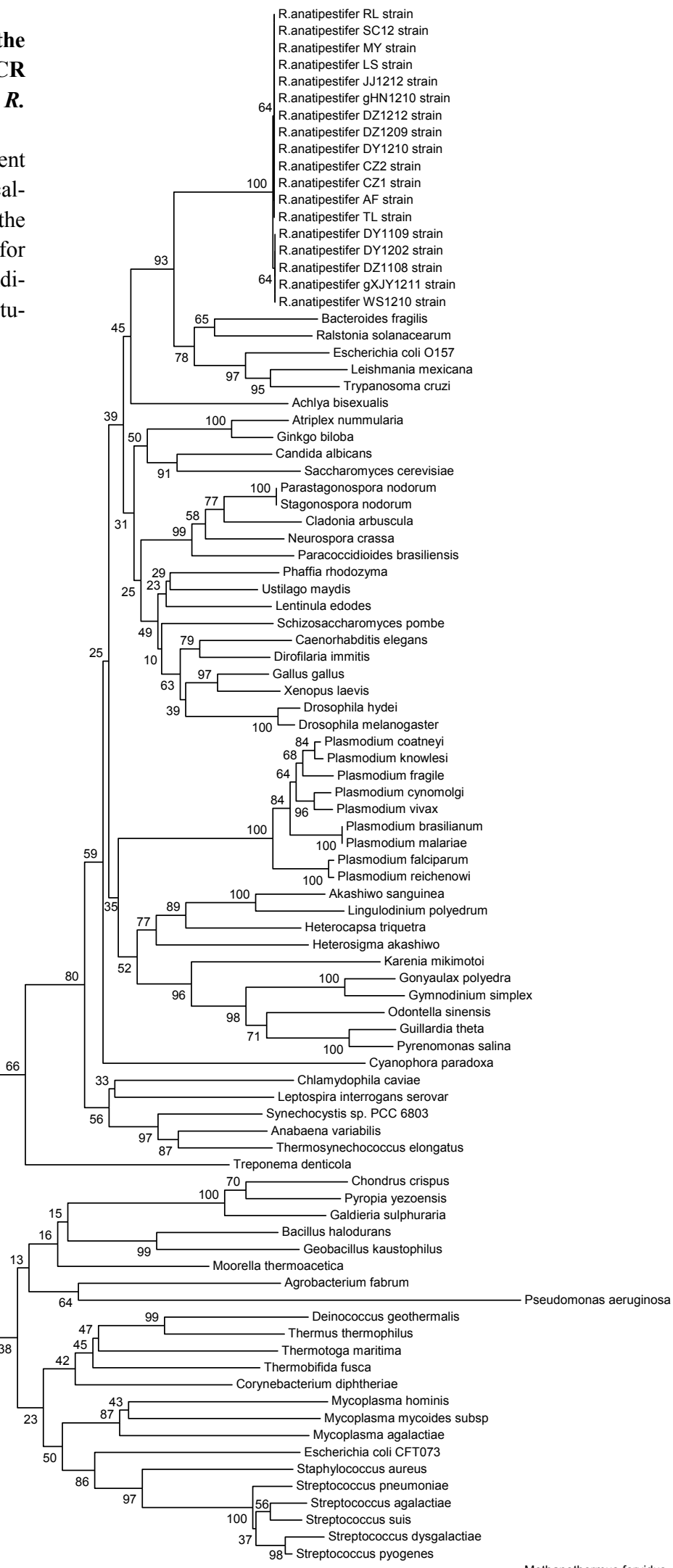

R.anatipestifer RL strain

R.anatipestifer SC12 strain

Ranatipestifer LS strain

anatipestifer gHN1210 strain

Ratipestifer DZ1212 strain

estifer DY1210 strain

R.anatipestifer CZ2 strain

R.anatipestifer CZ1 stran

R.anatipestifer DY1109 strai

(1)

anatipestifer gXJY1211 strai

anatipestifer WS1210 strain 
(a)

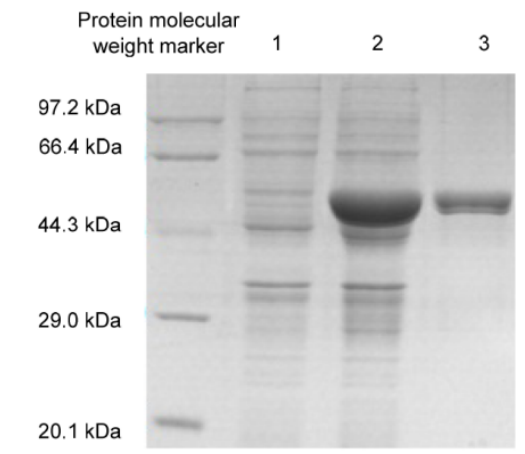

(b)
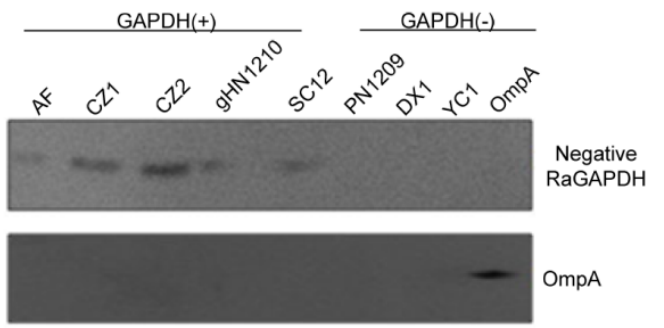

(c)

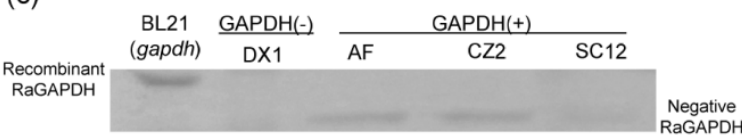

Fig. 4 SDS-PAGE analysis of recombinant RaGAPDH (a) and Western-blot analysis of RaGAPDH secretion (b) and recombinant RaGAPDH (c)

(a) SDS-PAGE (10\%) of the recombinant RaGAPDH produced in E. coli BL21 (DE3). Lane 1: total proteins extracted from uninduced cells carrying expression vector; Lane 2: total proteins extracted from induced cells with $0.5 \mathrm{mmol} / \mathrm{L}$ IPTG at $37{ }^{\circ} \mathrm{C}$ for $3 \mathrm{~h}$; Lane 3: soluble recombinant RaGAPDH fractions after Ni-NTA affinity chomatography. (b) Western-blot analysis of RaGAPDH secretion. Aliquots of $30 \mathrm{ml}$ of the supernatant cultures were precipitated with TCA and analyzed by Western-blot using anti-RaGAPDH specific antibodies, or anti-OmpA as a control of cytosolic contamination. The representative positive strains (AF, CZ1, CZ2, gHN1210, and SC12) and negative strains (PN1209, DX1, and YC1) were tested for extracellular RaGAPDH. (c) Western-blot analysis of recombinant RaGAPDH in E. coli BL21 (gapdh) and native RaGAPDH in cell-free supernatants of $R$. anatipestifer DX1, AF, CZ2, and SC12 strains

\subsection{Binding of purified RaGAPDH to host proteins}

The binding of purified RaGAPDH to host proteins including actin, fibrinogen, plasminogen, and fibronectin was analyzed by solid-phase ligandbinding assays. These proteins were coated on 96-well ELISA plates $(0.5 \mu \mathrm{g} /$ well $)$ and incubated with different concentrations of GAPDH $(0.125,0.25$,
$0.5,1.0,1.5,2.0$, and $2.5 \mu \mathrm{g} / \mathrm{ml})$. The amount of RaGAPDH bound to host proteins was determined as described in Section 2.8. The results indicated that RaGAPDH interacted with plasminogen and fibrinogen, but not with actin or fibronectin (Fig. 5).

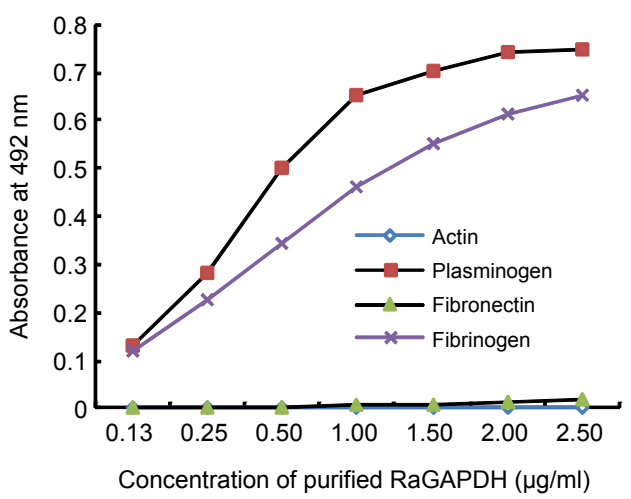

Fig. 5 Binding of purified RaGAPDH to host proteins by solid-phase ligand-binding assay

Actin, plasminogen, fibronectin, and fibrinogen were coated on 96-well microtiter plates and reacted with different concentrations of purified RaGAPDH. Data are presented as mean from three independent experiments

\section{Discussion}

As a cytoplasmic housekeeping enzyme with no detectable secretion or retention signal, GAPDH has been studied not only as a classical protein for its role in glycolysis but also as a model protein for enzyme kinetic analyses, crystallographic modeling, as well as for gene isolation and characterization. A large number of reports have shown that these enzymes are present on the surface of microbial pathogens, where they exert functions related to adhesion and/or virulence (Pancholi and Chhatwal, 2003). However, most studies are limited to fungi and Gram-positive pathogens, and there are few studies on Gram-negative bacteria besides $E$. coli. This is the first report documenting GAPDH activity in $R$. anatipestifer. Our results indicated that RaGAPDH is secreted by the Gram-negative $R$. anatipestifer, which is exposed and exported on the surface of the cell. The presence in the extracellular medium of RaGAPDH, but not OmpA, rules out cell lysis to explain this extracellular localization. Extracellular secretion of proteins is the major mechanism by which pathogens communicate with and intoxicate host cells. Previous reports showed that GAPDH presented on the surface of $S$. 
pyogenes was able to bind several mammalian proteins including the urokinase-type plasminogen activator receptor (uPAR/CD87) membrane protein on pharyngeal cells (Lottenberg et al., 1992; Pancholi and Fischetti, 1992; Winram and Lottenberg, 1996; Jin et al., 2005), regulate intracellular host-cell signaling events (Pancholi and Fischetti, 1997), and contribute to host immune evasion (Terao et al., 2006). Therefore, the secretion and association with the cell membrane of RaGAPDH might have biotechnological implications that are worth further investigation.

Members of the GAPDH family are grouped into three classes, and different microbial pathogens belong to different classes. $\mathrm{NAD}^{+}$-dependent GAPDH is the most common form and is usually located in the cytoplasm. In EHEC, gapA and gapC genes encode GAPDH proteins with highly similar sequences, and the gapA gene accounted for the exported GAPDH enzyme (Egea et al., 2007). In the Pseudomonas syringae genome, there are three paralogous gapdh genes encoding distinct GAPDHs, namely two class I enzymes having different molecular mass subunits and one class III biofunctional D-erythose-4-phosphate dehydrogenase/GAPDH enzyme (Elkhalfi et al., 2013). However, our results showed that there were several $R$. anatipestifer strains which did not have a homologous amplicon of GAPDH and showed no extracellular GAPDH activity. Whether lack of RaGAPDH in these strains is linked to attenuated virulence remains to be studied. Bioinformatics analysis of $R$. anatipestifer references genome, ATCC11845, RA-CH-1, RA-GD, however, showed the presence of only one copy of the gapdh gene. In addition, the PCR and sequence analysis results showed that there were one or two gapdh-like genes in $R$. anatipestifer DX1 and PN1209 strains. Therefore, we hypothesized that not all $R$. anatipestifer strains showed GAPDH activity, and there was a possibility that more than one gapdh-like gene existed in some $R$. anatipestifer strains, but not accounting for the extracellular GAPDH activity.

A recent proteomic study on $L$. monocytogenes showed classical cytosolic proteins like enolase that did not display a typical secretion signal, which could be exported through the SecA2-dependent secretion system (Lenz et al., 2003). Therefore, Gram-negative bacteria had developed one strategy consisting of vesicle-mediated export to enable such proteins to reach the extracellular space (Lenz et al., 2003). However, it is unclear whether GAPDH can be secreted in a similar way. Analysis of the RaGAPDH protein revealed its homology to the GAPDH family of glycolytic enzymes. There is no apparent signal sequence or transmembrane regions indicated by stretches of hydrophobic amino acid residues. However, it has a significant homology to the GAPDH proteins in EHEC, which had been shown to be membrane-associated and contain no recognized signal sequences. Our results also indicated that RaGAPDH was secreted by $R$. anatipestifer and its secretion mechanism needs to be further studied.

Attachment to host components is the critical first step in the establishment of infection by pathogens. Based on the putative role of extracellular RaGAPDH in R. anatipestifer infection, the ability of RaGAPDH to bind host proteins, known to interact with the extracellular GAPDH of other pathogens, was analyzed by solid-phase ligand-binding assays. We selected actin, fibrinogen, plasminogen, and fibronectin for this study. The results indicated that RaGAPDH interacted with plasminogen and fibrinogen, but not with actin or fibronectin. This is consistent with the fact that the C-terminal Lys residue of streptococcal GAPDH, important for plasminogen binding (Winram and Lottenberg, 1998), is conserved in the RaGAPDH sequence. Plasminogen is a serine protease that is abundant in extracellular fluids and associates with the surface of many cells in the body. Plasminogen-binding activity of extracellular bacterial GAPDH has been related to mechanisms of pathogenesis. Plasmin, the resulting activated form of plasminogen, can degrade extracellular matrix proteins and promote bacterial migration (Bergmann et al., 2004). In this way, the extracellular RaGAPDH, either exposed on the bacterial surface or secreted near the host cell, may contribute to the migration of these pathogens through digestive or respiratory tracts.

\section{Acknowledgements}

We thank Dr. Shao-bin SHANG at Northwestern University, China, for critical reading and suggestions for this manuscript.

\section{Compliance with ethics guidelines}

Ji-ye GAO, Cui-lian YE, Li-li ZHU, Zhi-ying TIAN, and Zhi-bang YANG declare that they have no conflict of interest.

This article does not contain any studies with human or animal subjects performed by any of the authors.

\section{References}

Aguilera, L., Ferreira, E., Gimenez, R., et al., 2012. Secretion of the housekeeping protein glyceraldehyde-3-phosphate 
dehydrogenase by the LEE-encoded type III secretion system in enteropathogenic Escherichia coli. Int. J. Biochem. Cell Biol., 44(6):955-962. [doi:10.1016/j.biocel. 2012.03.002]

Alvarez, A.H., Martinez-Cadena, G., Silva, M.E., et al., 2007. Entamoeba histolytica: ADP-ribosylation of secreted glyceraldehyde-3-phosphate dehydrogenase. Exp. Parasitol., 117(4):349-356. [doi:10.1016/j.exppara. 2007.04.016]

Bergmann, S., Rohde, M., Hammerschmidt, S., 2004. Glyceraldehyde-3-phosphate dehydrogenase of Streptococcus pneumoniae is a surface-displayed plasminogenbinding protein. Infect. Immun., 72(4):2416-2419. [doi:10. 1128/IAI.72.4.2416-2419.2004]

Chang, C.F., Hung, P.E., Chang, Y.F., 1998. Molecular characterization of a plasmid isolated from Riemerella anatipestifer. Avian. Pathol., 27(4):339-345. [doi:10. 1080/03079459808419349]

Colell, A., Ricci, J.E., Tait, S., et al., 2007. GAPDH and autophagy preserve survival after apoptotic cytochrome $\mathrm{c}$ release in the absence of caspase activation. Cell, 129(5):983-997. [doi:10.1016/j.cell.2007.03.045]

Crasta, K.C., Chua, K.L., Subramaniam, S., et al., 2002. Identification and characterization of CAMP cohemolysin as a potential virulence factor of Riemerella anatipestifer. J. Bacteriol., 184(7):1932. [doi:10.1128/JB.184.7.19321939.2002]

Daubenberger, C.A., Pöltl-Frank, F., Jiang, G., et al., 2000. Identification and recombinant expression of glyceraldehyde-3-phosphate dehydrogenase of Plasmodium falciparum. Gene, 246(1-2):255-264. [doi:10.1016/ S0378-1119(00)00069-X]

Egea, L., Aguilera, L., Gimenez, R., et al., 2007. Role of secreted glyceraldehyde-3-phosphate dehydrogenase in the infection mechanism of enterohemorrhagic and enteropathogenic Escherichia coli: interaction of the extracellular enzyme with human plasminogen and fibrinogen. Int. J. Biochem. Cell Biol., 39(6):1190-1203. [doi:10.1016/j.biocel.2007.03.008]

Eichenbaum, Z., Green, B.D., Scott, J.R., 1996. Iron starvation causes release from the group A streptococcus of the ADP-ribosylating protein called plasmin receptor or surface glyceraldehyde-3-phosphate-dehydrogenase. Infect Immun., 64(8):1956-1960.

Elkhalfi, B., Araya-Garay, J.M., Rodriguez-Castro, J., et al., 2013. Cloning and heterologous overexpression of three gap genes encoding different glyceraldehyde-3-phosphate dehydrogenases from the plant pathogenic bacterium Pseudomonas syringae pv. tomato strain DC3000. Prot. Expr. Purif., 89(2):146-155. [doi:10.1016/j.pep.2013.02. 005]

Figge, R.M., Schubert, M., Brinkmann, H., et al., 1999. Glyceraldehyde-3-phosphate dehydrogenase gene diversity in eubacteria and eukaryotes: evidence for intra- and inter-kingdom gene transfer. Mol. Biol. Evol., 16(4): 429-440. [doi:10.1093/oxfordjournals.molbev.a026125]

Hu, Q., Han, X., Zhou, X., et al., 2011. OmpA is a virulence factor of Riemerella anatipestifer. Vet. Microbiol., 150(3-4):278-283. [doi:10.1016/j.vetmic.2011.01.022]

Jin, H., Song, Y.P., Boel, G., et al., 2005. Group A streptococcal surface GAPDH, SDH, recognizes uPAR/
CD87 as its receptor on the human pharyngeal cell and mediates bacterial adherence to host cells. J. Mol. Biol., 350(1):27-41. [doi:10.1016/j.jmb.2005.04.063]

Kenny, B., Finlay, B.B., 1995. Protein secretion by enteropathogenic Escherichia coli is essential for transducing signals to epithelial cells. PNAS, 92(17): 7991-7995. [doi:10.1073/pnas.92.17.7991]

Leavitt, S., Ayroud, M., 1997. Riemerella anatipestifer infection of domestic ducklings. Can. Vet. J., 38(2):113.

Lenz, L.L., Mohammadi, S., Geissler, A., et al., 2003. SecA2-dependent secretion of autolytic enzymes promotes Listeria monocytogenes pathogenesis. PNAS, 100(21):12432-12437. [doi:10.1073/pnas.2133653100]

Ling, E., Feldman, G., Portnoi, M., et al., 2004. Glycolytic enzymes associated with the cell surface of Streptococcus pneumoniae are antigenic in humans and elicit protective immune responses in the mouse. Clin. Exp. Immunol., 138(2):290-298. [doi:10.1111/j.1365-2249.2004.02628.x]

Lottenberg, R., Broder, C.C., Boyle, M.D., et al., 1992. Cloning, sequence analysis, and expression in Escherichia coli of a streptococcal plasmin receptor. J. Bacteriol., 174(6):5204-5210.

Mavromatis, K., Lu, M., Misra, M., et al., 2011. Complete genome sequence of Riemerella anatipestifer type strain (ATCC 11845). Stand. Genomic Sci., 4(2):144-153. [doi:10.4056/sigs.1553865]

Modun, B., Williams, P., 1999. The staphylococcal transferrinbinding protein is a cell wall glyceraldehyde-3-phosphate dehydrogenase. Infect. Immun., 67(3):1086-1092.

Modun, B., Morrissey, J., Williams, P., 2000. The staphylococcal transferrin receptor: a glycolytic enzyme with novel functions. Trends Microbiol., 8(5):231-237. [doi:10. 1016/S0966-842X(00)01728-5]

Nagradova, N.K., 2001. Study of the properties of phosphorylating D-glyceraldehyde-3-phosphate dehydrogenase. Biochemistry (Moscow), 66(10):1067-1076. [doi:10.1023/A:1012472627801]

Pancholi, V., Fischetti, V.A., 1992. A major surface protein on group A streptococci is a glyceraldehyde-3-phosphatedehydrogenase with multiple binding activity. J. Exp. Med., 176(2):415-426. [doi:10.1084/jem.176.2.415]

Pancholi, V., Fischetti, V.A., 1997. Regulation of the phosphorylation of human pharyngeal cell proteins by group A streptococcal surface dehydrogenase: signal transduction between streptococci and pharyngeal cells. $J$. Exp. Med., 186(10):1633-1643. [doi:10.1084/jem.186.10. 1633]

Pancholi, V., Chhatwal, G.S., 2003. Housekeeping enzymes as virulence factors for pathogens. Int. J. Med. Microbiol., 293(6):391-401. [doi:10.1078/1438-4221-00283]

Parker, A.E., Bermudez, L.E., 2000. Sequence and characterization of the glyceraldehyde-3-phosphate dehydrogenase of Mycobacterium avium: correlation with an epidermal growth factor binding protein. Microb. Pathog., 28(3): 135-144. [doi:10.1006/mpat.1999.0335]

Pathanasophon, P., Sawada, T., Tanticharoenyos, T., 1995. New serotypes of Riemerella anatipestifer isolated from ducks in Thailand. Avian Pathol., 24(1):195-199. [doi:10. 1080/03079459508419059]

Pathanasophon, P., Phuektes, P., Tanticharoenyos, T., et al., 2002. A potential new serotype of Riemerella anatipestifer 
isolated from ducks in Thailand. Avian Pathol., 31(3): 267-270. [doi:10.1080/03079450220136576]

Schaumburg, J., Diekmann, O., Hagendorff, P., et al., 2004. The cell wall subproteome of Listeria monocytogenes. Proteomics, 4(10):2991-3006. [doi:10.1002/pmic.2004 00928]

Segers, P., Mannheim, W., Vancanneyt, M., et al., 1993. Riemerella anatipestifer gen. nov., comb. nov., the causative agent of septicemia anserum exsudativa, and its phylogenetic affiliation within the Flavobacterium-Cytophaga rRNA homology group. Int. J. Syst. Evol. Microbiol., 43(4): 768-776. [doi:10.1099/00207713-43-4-768]

Seifert, K.N., McArthur, W.P., Bleiweis, A.S., et al., 2003. Characterization of group B streptococcal glyceraldehyde3-phosphate dehydrogenase: surface localization, enzymatic activity, and protein-protein interactions. Can. J. Microbiol., 49(5):350-356. [doi:10.1139/w03-042]

Sirover, M.A., 2011. On the functional diversity of glyceraldehyde-3-phosphate dehydrogenase: biochemical mechanisms and regulatory control. Biochim. Biophys. Acta, 1810(8):741-751. [doi:10.1016/j.bbagen.2011.05.010]

Subramaniam, S., Chua, K.L., Tan, H.M., et al., 1997. Phylogenetic position of Riemerella anatipestifer based on 16S rRNA gene sequences. Int. J. Syst. Bacteriol., 47(2):562-565. [doi:10.1099/00207713-47-2-562]

Subramaniam, S., Huang, B., Loh, H., et al., 2000. Characterization of a predominant immunogenic outer membrane protein of Riemerella anatipestifer. Clin. Diagn. Lab. Immunol., 7(2):168-174. [doi:10.1128/CDLI. 7.2.168-174.2000]

Terao, Y., Yamaguchi, M., Hamada, S., et al., 2006. Multifunctional glyceraldehyde-3-phosphate dehydrogenase of Streptococcus pyogenes is essential for evasion from neutrophils. J. Biol. Chem., 281(20):14215-14223. [doi:10.1074/jbc.M513408200]
Tsai, H.J., Liu, Y.T., Tseng, C.S., et al., 2005. Genetic variation of the ompA and 16S rRNA genes of Riemerella anatipestifer. Avian Pathol., 34(1):55-64. [doi:10.1080/ 03079450400025471]

Tunio, S.A., Oldfield, N.J., Ala'Aldeen, D.A., et al., 2010. The role of glyceraldehyde 3-phosphate dehydrogenase (GapA-1) in Neisseria meningitidis adherence to human cells. BMC Microbiol., 10(1):280. [doi:10.1186/14712180-10-280]

Winram, S.B., Lottenberg, R., 1996. The plasmin-binding protein Plr of group A streptococci is identified as glyceraldehyde-3-phosphate dehydrogenase. Microbiology, 142(8):2311-2320. [doi:10.1099/13500872-142-8-2311]

Winram, S.B., Lottenberg, R., 1998. Site-directed mutagenesis of streptococcal plasmin receptor protein (Plr) identifies the C-terminal Lys334 as essential for plasmin binding, but mutation of the plr gene does not reduce plasmin binding to group A streptococci. Microbiology, 144(8): 2025-2035. [doi:10.1099/00221287-144-8-2025]

Yuan, J., Liu, W., Sun, M., et al., 2011. Complete genome sequence of the pathogenic bacterium Riemerella anatipestifer strain RA-GD. J. Bacteriol., 193(11): 2896-2897. [doi:10.1128/JB.01185-10]

Yuan, J., Li, L., Sun, M., et al., 2013. Genome sequence of avirulent Riemerella anatipestifer strain RA-SG. Genome Announc., 1(2):e0021812. [doi:10.1128/genomeA.001 01-13]

Zheng, L., Roeder, R.G., Luo, Y., 2003. S phase activation of the histone H2B promoter by OCA-S, a coactivator complex that contains GAPDH as a key component. Cell, 114(2):255-266. [doi:10.1016/S0092-8674(03)00552-X]

Zhou, Z., Peng, X., Xiao, Y., et al., 2010. Genome sequence of poultry pathogen Riemerella anatipestifer strain RA-YM. J. Bacteriol., 193(5):1284-1285. [doi:10.1128/ JB.01445-10]

\section{中文概要:}

本文题目：鸭疫里默氏杆菌的 GAPDH 同源体:一种有生物活性的胞外蛋白

A homolog of glyceraldehyde-3-phosphate dehydrogenase from Riemerella anatipestifer is an extracellular protein and exhibits biological activity

研究目的：对鸭疫里默氏杆菌的三磷酸甘油醛脱氢酶（GAPDH）进行鉴定和生物学特征分析。

创新要点: 首次证实鸭疫里默氏杆菌具有 GAPDH 的同源体酶（RaGAPDH）是一种无信号肽和跨膜区的胞外蛋白酶, 具有将 3-磷酸甘油醛转化为 1,3 -二磷酸甘油酸的活性, 可与纤维蛋白溶酶原及纤维蛋白原发生结合, 推测 该酶可能是鸭疫里默氏杆菌的一个新发现的毒力因子。

研究方法：1. 对分离自重庆、四川地区的鸭疫里默氏杆菌（表 1）菌体细胞表面蛋白（图 1a）和 CZ2、SC12、YC1 三株菌胞外蛋白（图 1b）的 GAPDH 活性进行检测，对其编码基因进行 PCR 鉴定（图 2）和克隆测序分析 （图 3）；2．采用染色体步移技术获得 CZ2 的 GAPDH 编码基因进行原核表达（图 $4 \mathrm{a}$ 和 4c ）；3．以获得 的具有活性的重组 GAPDH 为抗原, 制备鼠原多克隆抗体并采用 Western-blot 方法对鸭疫里默氏杆菌的胞外 分泌蛋白进行检测分析（图 4b）；4. 采用固相配体结合试验检测 RaGAPDH 与纤维蛋白溶酶原、血纤维 蛋白原、肌动蛋白和纤连蛋白的结合作用（图 5)。

重要结论: 鸭疫里默氏杆菌具有三磷酸甘油醛脱氢酶同源体, 具有 GAPDH 活性, 能与纤维蛋白溶酶原和血纤维蛋白 原结合, 可能是其重要的毒力因子。

关键词组: 鸭疫里默氏杆菌; 三磷酸甘油醛脱氢酶; 胞外蛋白 